\title{
The major-axis effect: Axes of bilateral symmetry or loci of neural interactions?
}

\author{
ALAN A. HARTLEY \\ University of California, Irvine, California 92717
}

\begin{abstract}
Distortions in perceptual judgments have been attributed to the axes of bilateral symmetry of surrounding figures. In the figures used in prior investigations, the axes of bilateral symmetry have been confounded with loci where heightened neural interaction might be expected to occur. In the present experiment, subjects reproduced dots surrounded by figures for which the axes of symmetry and loci of hypothesized neural interactions differed. The observed displacements supported the hypothesis that distortions were due to spreading neural effects of the surrounding figures rather than to the effects of axes of bilateral symmetry.
\end{abstract}

In a series of experiments, Wenderoth has demonstrated that settings of gravitational vertical are systematically displaced toward the major axes of surrounding figures whether these axes are physically present lines or implicit, virtual lines (Beh \& Wenderoth, 1972; Beh, Wenderoth, \& Purcell, 1971; Wenderoth, 1973; Wenderoth \& Curthoys, 1974). The major axes of a figure are those which divide the figure into mirror images, the axes of bilateral symmetry. Wenderoth (1973) has suggested that the major axis effect may be due to inhibitory interactions between cortical orientation detectors. This possibility will be termed the neural interaction hypothesis. Wenderoth and Curthoys (1974) reject that hypothesis, arguing that axes of symmetry have emergent properties which cannot be accounted for by simple neural events. This alternative will be termed the bilateral symmetry hypothesis. Consider an acute angle. The line bisecting the angle is both an axis of bilateral symmetry and the locus at which lateral inhibition from the lines comprising the angle would summate to give greater inhibition than surrounding points. For most of the figures used to investigate the major-axis effect, the loci of presumed heightened neural interaction and the axes of bilateral symmetry have been identical (but cf. Wenderoth \& Curthoys, 1974, Experiment II). A sampling of the figures is shown in Figure 1. The present experiment investigates figures in which the loci of neural interaction and the axes of bilateral symmetry are not the same in order to separate their effects on perceptual judgments. The figures used in the present experiment are shown in Figure 2.

The loci of neural interactions shown in Figure 2

I am grateful to Jay Brinegar for his assistance in data coding and analysis and to Joellen Hartley for suggestions during the preparation of this report. Requests for reprints should be addressed to the author who is now at the Department of Psychology, Scripps College, Claremont, California 91711. need not be due to lateral inhibition, nor need they be due to cortical rather than retinal interaction. It is only necessary to assume that real lines give rise to neural effects that spread away from their borders.

The bilateral symmetry hypothesis predicts that the apparent location of a point within the boundaries of the figures should be displaced toward a real line, a figural contour, or an implicit line, an axis of bilateral symmetry, as shown in Figure 2, whichever is closer to the point. The neural interaction hypothesis predicts that points should only be affected by surrounding physically present lines. Whether the locations are displaced toward the lines or away from them depends on whether excitatory or inhibitory effects are posited. The bilateral symmetry hypothesis predicts that points falling on a virtual contour will show no displacement whatever. The neural interaction hypothesis predicts that, even after displacement, points lying on a line of heightened neural interaction will still lie on that line. For example, points along the diagonal of the square may appear closer to or farther from the corner of the square, but they will remain equidistant from the borders of the square.
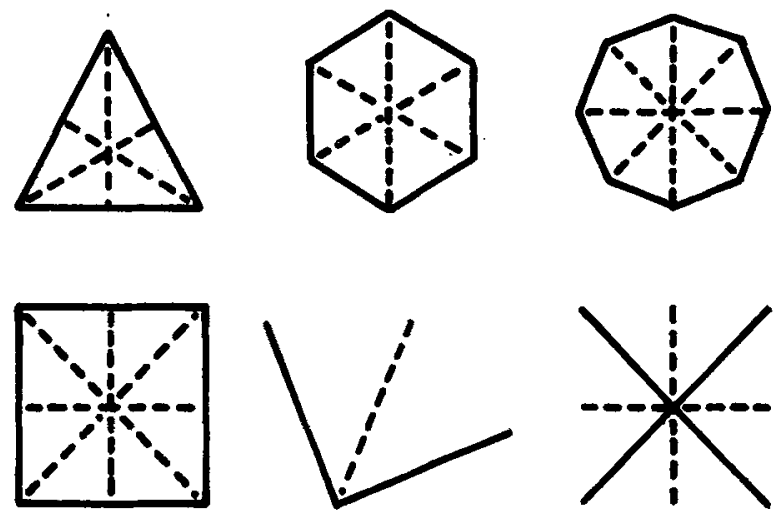

Figure 1. Stimuli used in investigations of the major-axis hypothesis. Major axes are shown by dashed lines. 

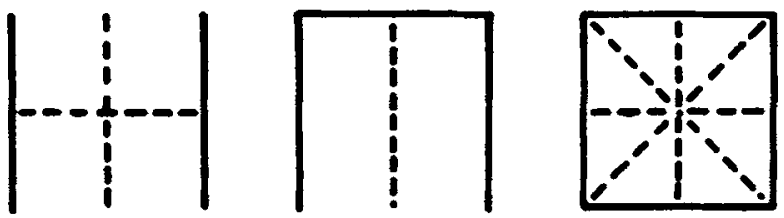

b
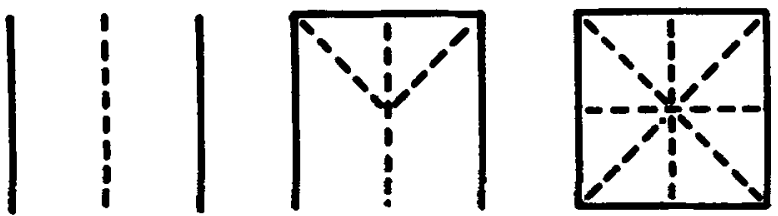

Figure 2. Stimuli used in the present study. (a) Axes of bilateral symmetry shown by dashed lines. (b) Loci of neural interactions shown by dashed lines.

\section{METHOD}

\section{Stimuli and Procedure}

Each stimulus presentation consisted of one of the figures shown in Figure 2 printed in black, centered on a sheet of heavy, white paper, $8.5 \mathrm{in}$. $(21.59 \mathrm{~cm})$ square. The lines of the figures were $12 \mathrm{~cm}$ in length and $1 \mathrm{~mm}$ in width. In addition, a dot, $1 \mathrm{~mm}$ in diameter, was drawn on the sheet using India ink. Both the horizontal and vertical locations of the dots were varied. Five horizontal locations were used: at the point midway between the two vertical lines, 2 or $4 \mathrm{~cm}$ to the left of the midway point, or 2 or $4 \mathrm{~cm}$ to the right of the midway point. The five vertical locations used were analogous to the horizontal locations. Combining the five horizontal and five vertical positions, there were 25 locations in which the dot could appear. Each of these 25 locations was presented to the subject once with each of the three figures to yield 75 experimental conditions.

The subject's task on each trial was to take a stimulus sheet and place it in a heavy, pasteboard frame that was attached to the table in front of him or her. The subject then took a test sheet, a blank sheet of paper $8.5 \mathrm{in}$. $(21.59 \mathrm{~cm})$ square, and placed it in a second frame. The frame for the test sheet was placed either $5 \mathrm{~cm}$ to the left or to the right of the frame for the stimulus sheet. The two frames were placed at different distances from the subject: either the upper border of the test frame was $14 \mathrm{in} .(35.56 \mathrm{~cm})$ from the edge of the table and the upper border of the stimulus frame $10 \mathrm{in.}(25.40 \mathrm{~cm})$ or vice versa. The stimulus and reproduction sheets were viewed at angles ranging, approximately, from $40^{\circ}-50^{\circ}$ to $70^{\circ}-90^{\circ}$ below the straight-ahead. The four possible arrangements of the frames were counterbalanced over subjects.

After viewing the stimulus sheet for as long as desired, the participant, using a pencil, attempted to reproduce the position of the dot on the blank test sheet. If the subject was unsatisfied with the reproduced dot, the test sheet was replaced with a blank one and the reproduction was repeated. The subject was asked to keep his or her head in a steady position throughout the procedure.

The reproductions were made in blocks of 25 sheets, all with the same figure. The order in which the 25 dot locations were presented within each block was randomly determined. The order of figures was counterbalanced across subjects. The procedure was self-paced and required 15 to $30 \mathrm{~min}$.

\section{Subjects}

Twelve students, eight female and four male, from a course in methods of psychological research volunteered to participate in partial fulfillment of a course requirement. The students were right-handed and had normal or corrected-to-normal vision.

\section{RESULTS AND DISCUSSION}

For each reproduction, the difference between the reproduced and actual location of the dot was determined both in the horizontal and vertical directions. The locations of the dots on the stimulus figures and on the reproductions were obtained by laying a millimeter-ruled grid over the sheet and recording the coordinates of the center of the dot to the nearest $1 / 2 \mathrm{~mm}$. When a dot was reproduced to the right of or above the actual position, the discrepancy was designated as positive; otherwise, it was designated as negative.

An analysis of variance carried out on the horizontal discrepancies indicated only a significant effect of the horizontal location of the dot, the "column" in which it appeared, $F(4,44)=14.452, \mathrm{p}<.01$. Points midway between the vertical lines of the figures showed no horizontal displacement. Dots in other columns were displaced toward the nearest, physically present vertical line. The mean displacements are given in Table 1. A planned comparison showed that displacements were significantly greater for points $4 \mathrm{~cm}$ from the lines of the figure than for points $2 \mathrm{~cm}$ away, $t(11)=2.391, p<.05$.

Analysis of the vertical displacements yielded significant main effects of the figure, $F(3,33)=7.653$, $\mathrm{p}<.01$, and of the vertical location of the dot, the "row" in which it appeared, $F(4,44)=5.295$, $\mathrm{p}<.01$. Overall displacement in the figure consisting of two parallel, vertical lines was downward; for the three-sided, box figure, displacement was upward; and for the square, displacement was negligible. Over all three figures, dots in the three rows closest to the top of the stimulus display were displaced upward; those in the lower two rows were displaced toward the bottom of the display. Average vertical displacements as a function of figure and row are given in Table 1. Small, but significant, interaction effects were found for Figure by $\operatorname{Row}, F(8,88)=2.061$, $p<.05$, and for Figure by Column, $F(8,88)=2.214$, $\mathrm{p}<.05$. The average displacements for all points are displayed in Figure 3.

The results indicate that perceptual judgments are affected by the locations where neural interactions are presumed to occur rather than by axes of bilateral symmetry. Dots which did not lie on hypothesized loci of interactions were displaced toward the nearest physically present line; there was no component of displacement toward the virtual lines. This can be seen most clearly in the displacement of the columns of dots. If the columns are numbered sequentially from the left, then the bilateral symmetry hypothesis predicts that dots in Columns 1 and 5 should be displaced toward the borders of the figure while dots in 
Table 1

Deviations from Correct Reproduction

\begin{tabular}{|c|c|c|c|c|c|c|c|c|c|c|}
\hline & \multicolumn{3}{|c|}{ Horizontal Deviation ( $\mathrm{mm}$ ) } & \multicolumn{7}{|c|}{ Vertical Deviation (mm) } \\
\hline & & Mean & $S D$ & & & Mean & SD & Figure & Mean & $\mathrm{SD}$ \\
\hline & -4 & -4.08 & 2.12 & & -4 & -3.19 & 2.49 & Two-Sided & 2.26 & 2.46 \\
\hline Horizontal & -2 & -6.17 & 2.41 & Vertical & -2 & -4.04 & 2.63 & Three-Sided & 1.86 & 2.65 \\
\hline Location* & 0 & .01 & 1.89 & Location $\dagger$ & 0 & 1.26 & 2.50 & Four-Sided & -.24 & 3.09 \\
\hline \multirow[t]{2}{*}{ ("Column") } & +2 & 5.70 & 1.97 & ("Row") & +2 & 3.33 & 2.90 & & & \\
\hline & +4 & 2.93 & 1.97 & & +4 & 1.58 & 2.85 & & & \\
\hline
\end{tabular}

*Centimeters from vertical midline

tCentimeters from horizontal midline

Columns 2 and 4 should be displaced toward the vertical midline of the figure. The observed displacement in all four columns was in the direction of the nearest border. The absence of displacement toward axes of bilateral symmetry may also be seen in the rows of the two-line figure. If the rows are numbered sequentially from top to bottom, then dots in the second row should have been reproduced lower than their actual position and dots in the fourth row should have been reproduced higher, showing displacement toward the axis of symmetry along the horizontal midline of the figure. The observed displacement in the second row was slightly upward $\overline{\mathrm{X}}=.90, \mathrm{SD}=3.05$ ) and, in the fourth row, it was downward $(\overline{\mathrm{X}}=-5.97, \mathrm{SD}=2.71)$. The neural interaction hypothesis would predict only horizontal displacement in the two-line figure. The overall downward displacement may have been an artifact of the viewing angle, as discussed below.

Dots presented at the loci of interactions were displaced from their true positions when reproduced, but the direction of displacement was generally along the implicit contour that would be formed by neural interactions rather than toward surrounding lines. The three-line figure affords the clearest contrast between the neural interaction and bilateral symmetry hypotheses for points lying on a locus of interaction but not on an axis of symmetry. The neural interaction hypothesis predicts that the dot located at Row 2, Column 2 should be displaced along the negative diagonal. The bilateral symmetry hypothesis predicts that displacement should be horizontal. Analyses of the direction of displacement showed that the mean direction was not significantly different from $135^{\circ}$ (measured counterclockwise from horizontal),
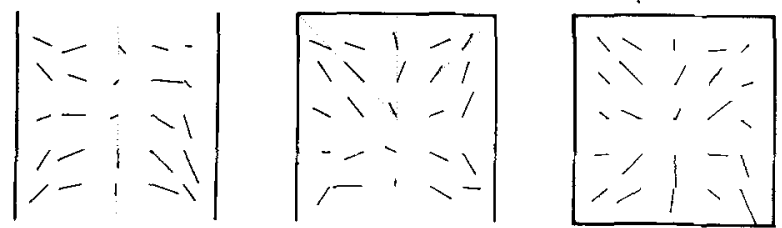

Figure 3. Magnitude and direction of observed displacements. (For clarity, magnitudes have been doubled relative to the rest of the figure.) $t(11)=.179$, n.s., but did differ significantly from the horizontal, $t(11)=3.92, p<.01$. Similarly, the neural interaction hypothesis predicts that the dot located at Row 2, Column 4 should be displaced along the positive diagonal while the bilateral symmetry hypothesis predicts horizontal displacement. The mean direction of displacement did not differ significantly from $45^{\circ}$ (counterclockwise from horizontal), $t(11)=1.321$, n.s., but did differ from the horizontal, $\mathrm{t}(11)=3.967, \mathrm{p}<.01$.

Vertical displacement was affected by the presence of horizontal lines in the figures, reflecting movement of the reproduced locations toward the lines: Addition of the horizontal bar in the three-sided figure caused an upward shift in the reproduced positions relative to the two-line figure; the bilateral symmetry hypothesis predicted only horizontal displacement, since the only virtual line is vertical. Introduction of a second horizontal bar to form a square negated the upward displacement seen in the three-sided figure.

Dots were displaced horizontally toward the nearer of the two vertical lines. The displacement was greater for dots located $4 \mathrm{~cm}$ from the line than for those $2 \mathrm{~cm}$ from the line. This result agrees with those of previous investigations of perceptual judgments of locations within extents or areas comparable in size to those used in this experiment. Asked to estimate the distance from a line to a marker as a fraction of the distance from the line to a second line, subjects overestimate the distance between the marker and the nearer line for most marker positions (Carr \& Garner, 1952; Chapanis \& Leyzorek, 1950; Levett, 1952; Miller, 1950). For locations close to a line, the distance is underestimated (except for very small distances, which are overestimated). If apparent distances are increased (or decreased for short distances) by the presence of a line, then a subject asked to produce a marker at a specified fraction of the distance from one line to another should place the marker closer to (farther from) the nearest line than it should actually be so as to make it appear to be the requested distance. This is the result that is observed both for production of interpolations along a line (Nash, 1964) and for reproductions of locations within a bordered area that have been presented for 
inspection and then removed (Attneave, 1955; Taylor, 1961). Lederman and Taylor (1969) found this result when subjects reproduced in pencil the location of either a seen or a felt spot on a $3 \times 5$ in. card.

To explain findings such as these, Deutsch (1964) argues that lateral inhibition from a line will lower the excitability of points lying between a particular location near the line and the line itself. This will lead to an overestimation of the distance between the location and the line. The spread of excitation from the line, perhaps due to nystagmic eye movements, should cause the distance between a line and a point close to it to be underestimated; that is, the apparent location should be displaced toward the line. (Also see Ganz, 1966.) Thus the horizontal displacements of reproduced dot positions observed in the present experiment are compatible with the predictions of the lateral inhibition model.

The model does not account for the tendency for locations in the two-line figure to be reproduced below their actual position. There is no ready explanation for this finding which has also been reported by Attneave (1955) and Nash (1964). It may be due to the steep angles at which the subjects viewed the stimuli and reproductions.

Studies requiring the subject to set a rod to the gravitational vertical find that the settings deviate toward a virtual line when that is the nearest feature. The present study found that reproduced dots tended to be displaced away from virtual lines and toward physically present lines. Thus the possibility remains that judgments of the vertical in the context of a surrounding figure are affected by the axes of bilateral symmetry of the figure, while other judgments in the same context are affected by loci of neural interaction. Wenderoth and Curthoys (1974) have argued that vertical settings involve a psychological component that cannot be accounted for completely by simple neural mechanisms.

The neural interaction hypothesis proposed here is formally isomorphic to a geometry proposed by Blum (1973) as a basis for the analysis of biological shape. Blum proposed two models. In each, the contours of a figure are assumed to give rise to axes of symmetry (sym-axes). Although Blum gives a rigorous mathematical development, the locations of sym-axes can be arrived at intuitively. For the first model, imagine that a grassfire is set along the borders of the figure. The sym-axes of the figure are the loci where the fires from different borders are quenched. For the second model, imagine that the borders generate waves in a fluid medium. The loci of standing waves are the sym-axes. Since waves can pass through one another while fires cannot, the "pond" model gives rise to sym-axes in addition to those of the "grassfire" model. The loci of neural interactions in the present discussion are identical to the sym-axes of Blum's "pond" model. Blum arrived at his geometry by searching for a way to describe shape that would capture the fundamental similarities among superficially different figures. No consideration was given to the way in which a perceptual system could carry out such an analysis. The neural interaction hypothesis was arrived at by making an assumption about the way in which contours might be represented in perception and, from that, deducing that interactions would take place at specified loci between contours, providing a mechanism for the extraction of shape descriptions called for in Blum's geometry.

The present experiment cannot separate the "pond" and "grassfire" models. Psotka (Note 1), however, found that results of a task in which subjects freely placed a dot anywhere within a figure agreed with the "grassfire" model.

Andrews (1967; Andrews, Butcher, \& Buckley, 1973; Andrews, Webb, \& Miller, 1974) has argued that, for positions separated by more than 10 to $30 \mathrm{~min}$ of arc, the distance between features is signaled by inefficient, higher-order neural units. The inefficiency in length comparisons was found to increase as the length increased (Andrews et al., 1974). This efficiency hypothesis may provide an alternative explanation for the present results. A person asked to reproduce the position of a dot could construct an implicit line from the nearest edge, stopping when the length was not discriminably different from the analogous implicit line on the stimulus sheet. The reproduced dot would be displaced toward the edge and the magnitude of the displacement would increase as the distance of the dot from the edge increased. This is what was found in the present experiment, and it agrees closely with the results of Taylor (1961) and Lederman and Taylor (1969) using white cards with no features other than the dot as stimuli. There are two exceptions to the movement toward the edges. First, dots placed very close to the edges in those earlier studies were not displaced toward them. It may be that, at such small separations, efficient, lower-order units take over. Second, in those studies and in the present, dots placed midway between two edges did not move toward either edge. It may be that bisection is a separate operation, or it may be that the selected point must satisfy two sets of positional constraints.

Andrews et al. (1973) also found that gaps in a linear extent further reduced the efficiency with which position was determined. The lines of the figures in the present study may have functioned as gaps. The decreased efficiency, then, may be reflected in the displacements attributable to the figures. The predictions of the efficiency hypothesis are identical to those of the locus of neural interaction hypothesis. 
Under the efficiency explanation, however, the presumed loci of neural interaction would be points of stability, not because of any neural events taking place there, but because of constraints on judgment.

The disruptiveness of gaps underscores a peculiar implication of the present results. The distance between the dot and the nearest edge of the paper may be thought of as an extent that is filled on the stimulus sheet by a border of the figure but that is empty on the reproduction sheet. The usual finding is that a filled extent is judged longer than an unfilled extent of the same length (Robinson, 1972). A person attempting to match a filled space, then, should produce an extent greater than the actual length. In the present study, the produced lengths were less than the actual length, as the efficiency explanation predicts, indicating that the observed displacements cannot be attributed to factors that produce the filled and empty space illusion. Models predicting that illusion must be ruled out as explanations for the present results. Such models include those postulating comparative judgment based on information integration (Clavadetscher \& Anderson, 1977) or contextual effects of frame size (Brigell, Uhlarik, \& Goldhorn, 1977).

Because the stimulus and reproduction sheets were separated, it was not possible to focus on both simultaneously. Hence, another alternative explanation of the present results is that they were due to the representation of the perceptual experience in memory. Corners, edges, and boundaries may serve as salient anchor points for encoding, and their salience may be increased or modified when the location of the dot is decoded (also see Attneave, 1955; Taylor, 1961).

\section{REFERENCE NOTE}

1. Psotka, J. Perceptual processes that create stick figures and balance. Unpublished manuscript: University of Waterloo, 1977.

\section{REFERENCES}

ANDREws, D. P. Perception of contour orientation in the central fovea. Part II: Spatial integration. Vision Research, 1967, 7, 999-1013.

ANdrews, D. P., Butcher, A. K., \& Buckley, B. R. Acuities for spatial arrangement in line figures: Human and ideal observers compared. Vision Research, 1973, 13, 599-620.
ANDRews, D. P., WegB, J, M., \& Miller, D. T. Acuity for length comparison in continuous and broken lines. Vision Research, 1974, 14, 757-766.

Attmeave, F. Perception of place in a circular field. American Journal of Psychology, 1955, 68, 69-82.

BEh, H. C., \& WeNDERoth, P. M. The effect of variation of frame shape on the angular function of the rod-and-frame illusion. Perception \& Psychophysics, 1972, 11, 35-37.

Beh, H. C., Wenderoth, P. M., \& Purcell, A. T. The angular function of the rod-and-frame illusion. Perception \& Psychophysics, 1971, 9, 353-355.

BuUm, H. Biological shape and visual science (Part 1). Journal of Theoretical Biology, 1973, 38, 205-287.

Brigell, M., Uhlarik, J., \& Goldhorn, P. Contextual influences on judgments of linear extent. Journal of Experimental Psychology: Human Perception and Performance, 1977, 3, 105-118.

CarR, W. J., \& Garner, W. R. The maximum precision of reading fine scales. Journal of Psychology, 1952, 34, 85-94.

Chapanis, A., \& Leyzorek, M. Accuracy of visual interpolation between scale markers as a function of the number assigned to the scale interval. Journal of Experimental Psychology, $1950,40,655-667$.

Clavadetscher, J. E., \& Anderson, N. H. Comparative judgment: Tests of two theories using the Baldwin figure. Journal of Experimental Psychology: Human Perception and Performance, 1977, 3, 119-135.

Deursch, J. A. Neurophysiological contrast phenomena and figural aftereffects. Psychological Review, 1964, 71, 19-26.

GANZ, L. Mechanism of the figural aftereffects. Psychological Review, 1966, 73, 128-150.

Lederman, S. J., \& TaYlor, M. M. Perception of interpolated position and orientation by vision and active touch. Perception \& Psychophysics, 1969, 6, 153-159.

LEVETT, C. M., JR. Errors of interpolation in instrument reading and setting. Journal of Applied Psychology, 1952, 36, 49-52.

MilleR, H. K., JR. An exploratory study of linear interpolation. Journal of Applied Psychology, 1950, 34, 367-370.

$\mathrm{N}_{\mathrm{A} S \mathrm{H}}, \mathrm{H}$. The judgment of linear proportions. American Journal of Psychology, 1964, 77, 480-484.

RoBinson, J. O. The psychology of visual illusion. London: Hutchinson, 1972.

TAYLOR, M. M. Effect of anchoring and distance perception on the reproduction of forms. Perceptual and Motor Skills, 1961, 12, 203-230.

Wenderoth, P. M. The effects of tilted outline frames and intersecting line patterns on judgments of vertical. Perception \& Psychophysics, 1973, 14, 242-248.

WeNDERoth, P. M., \& Curthoys, I. On the non-additivity of visual illusions. Quarterly Joumal of Experimental Psychology, $1974,26,549-555$.

(Received for publication November 17, 1977; revision accepted March $17,1978$. 